\title{
Is moderate hypofractionation accepted as a new standard of care in north america for prostate cancer patients treated with external beam radiotherapy? Survey of genitourinary expert radiation oncologists
}

\author{
Shearwood McClelland III 1,2, Kiri A. Sandler ${ }^{3}$, Catherine Degnin ${ }^{4}$, Yiyi Chen ${ }^{4}$, Arthur Y. Hung ${ }^{2}$, Timur Mitin ${ }^{2}$ \\ ${ }^{1}$ Department of Radiation Oncology, Indiana University School of Medicine, Indianapolis, IN, U.S.A; ${ }^{2}$ \\ Department of Radiation Medicine, Oregon Health and Science University, Portland, OR, U.S.A; ${ }^{3}$ Department \\ of Radiation Oncology, University of California at Los Angeles, Los Angeles, CA, U.S.A; ${ }^{4}$ Biostatistics \\ Shared Resource, Oregon Health and Science University, Portland, OR, U.S.A
}

\section{ABSTRACT}

Introduction: Several recent randomized clinical trials have evaluated hypofractionated regimens against conventionally fractionated EBRT and shown similar effectiveness with conflicting toxicity results. The current view regarding hypofractionation compared to conventional EBRT among North American genitourinary experts for management of prostate cancer has not been investigated.

Materials and Methods: A survey was distributed to 88 practicing North American GU physicians serving on decision - making committees of cooperative group research organizations. Questions pertained to opinions regarding the default EBRT dose and fractionation for a hypothetical example of a favorable intermediate - risk prostate cancer (Gleason $3+4$ ). Treatment recommendations were correlated with practice patterns using Fisher's exact test.

Results: Forty - two respondents (48\%) completed the survey. We excluded from analysis two respondents who selected radical hypofractionation with 5 - 12 fractions as a preferred treatment modality. Among the 40 analyzed respondents, 23 (57.5\%) recommend conventional fractionation and 17 (42.5\%) recommended moderate hypofractionation. No demographic factors were found to be associated with preference for a fractionation regimen. Support for brachytherapy as a first choice treatment modality for low - risk prostate cancer was borderline significantly associated with support for moderate hypofractionated EBRT treatment modality $(\mathrm{p}=0.089)$.

Conclusions: There is an almost equal split among North American GU expert radiation oncologists regarding the appropriateness to consider moderately hypofractionated EBRT as a new standard of care in management of patients with prostate cancer. Physicians who embrace brachytherapy may be more inclined to support moderate hypofractionated regimen for EBRT. It is unclear whether reports with longer followups will impact this balance, or whether national care and reimbursement policies will drive the clinical decisions. In the day and age of patient - centered care delivery, patients should receive an objective recommendation based on available clinical evidence. The stark division among GU experts may influence the design of future clinical trials utilizing EBRT for patients with prostate cancer.

\section{ARTICLE INFO}

\section{Keywords:}

Prostatic Neoplasms; Dose

Hypofractionation; Neoplasm

Grading

Int Braz J Urol. 2019; 45: 273-87

Submitted for publication:

April 17, 2018

Accepted after revision:

September 13, 2018

Published as Ahead of Print: October 30, 2018 


\section{INTRODUCTION}

The standard eight-to-nine week course of conventional external beam radiation therapy (EBRT) for prostate cancer although effective, disrupts patients' normal lives, causes financial toxicity to patients and places a significant f1nancial strain on the healthcare system. For these reasons, hypofractionated radiation therapy (RT), which involves larger radiation doses administered over an overall shorter time period, has increased in popularity, and has been established in other disease sites, such as breast cancer, bone metastases, bladder cancer, glioblastoma and non - small cell lung cancer (1-5). Four randomized clinical trials have compared moderately fractionated regimens to conventionally fractionated RT in prostate cancer (Table-1) (6-11). With 5 -years of follow-up, none revealed inferiority of hypofractionation regarding the treatment outcomes, and the toxicity reports are contradictory, with no overwhelming and reproducible toxicity associated with a moderately hypofractionated regimens using 2.5 to 3 Gy per fraction. We sought to determine the current view of moderate hypofractionation among North American genitourinary (GU) radiation oncology experts due to their influence in shaping the direction of national guidelines.

\section{MATERIALS AND METHODS}

\section{Survey design and deployment}

The survey was designed to assess the opinions of GU experts on the default EBRT dose and fractionation for a hypothetical patient with a favorable - intermediate risk prostate cancer who would require by most current conventions EBRT to prostate alone without prophylactic irradiation of pelvic lymph nodes. Three fractionation schemes were offered as choices: conventional fractionation (78 Gy in 2 Gy fractions, 79.2 Gy in 1.8 Gy fractions or equivalent), moderate hypofractionation (70 Gy in 2.5 Gy fractions or equivalent), or SBRT / radical hypofractionation (5 - 12 fractions or equivalent). The study was approved by IRB and electronically sent to 88 North American GU oncology physicians, who ser- ve on cooperative group research organizations such as NRG Oncology. The survey was designed and hosted by Research Electronic Data Capture (REDCap), and contained screening questions to ensure respondents were currently practicing, not in training, and specializing in GU oncology (12). A copy of the survey is available in the Appendix 1.

\section{Statistical analysis}

Based on responses, participants were categorized as "supporters" or "opponents" of moderate hypofractionation. For the purposes of this study, only responders choosing conventional fractionation or moderate hypofractionation were included. Fisher's exact test was used to determine whether treatment recommendations were correlated with practice patterns. $\mathrm{R}(\mathrm{R}$ version 3.3.3 (2017-03-06)) was used for all data analysis. Statistical significance was set at $\mathrm{p}<$ 0.05 .

\section{RESULTS}

Forty - two of the 88 radiation oncologists completed the survey, of whom 40 (95.2\%) recommended either conventional fractionation or moderate hypofractionation; two $(4.8 \%)$ recommended stereotactic body radiation therapy (SBRT) (Figure-1) and were excluded from the analysis. Of 40 analyzable respondents, 23 $(57.5 \%)$ recommended conventional fractionation and 17 (42.5\%) recommended moderate hypofractionation.

No demographic factors (years in practice, geographic location of residency, geographic location of practice, monthly patient volume, practice type) as well as other clinical positions (active surveillance recommendation preference, brachytherapy boost advocacy, self-identification as an expert brachytherapist, likelihood of considering stereotactic body RT for oligometastatic disease, likelihood of prophylactically irradiating pelvic lymph nodes, support of advanced imaging techniques) were significantly associated with support of moderate hypofractionation. Only the choice of brachytherapy as a preferred 
treatment option for patients with low - risk prostate cancer approached significance for recommendation of hypofractionation $(p=0.089)$ (Table-2).

\section{DISCUSSION}

Biological considerations of a markedly lower alpha / beta ratio of prostate cancer in comparison to surrounding normal tissues led researchers to clinical investigation of hypofractionated regimens in management of patients with prostate cancer with EBRT (13). Four large international randomized clinical trials have established non - inferiority of moderate hypofractionation (2.5 - 3 Gy per fraction), with varying toxicity results, some supporting conventional, others hypofractionated regimens, but none reporting overwhelming toxicity within the 5 - years of a follow-up period (Table-1) (6-11).

The degree of acceptance / rejection of treatment modalities in North America is to a significant extent shaped by opinions of leading academic physicians who define and periodically update national treatment guidelines, author consensus statements and shape the future clinical trial protocols. Because of this influence, we sought to determine the acceptance of hypofractionation for prostate cancer among North American GU radiation oncology experts (14).

The results of this study indicate that hypofractionated EBRT, defined as 70 Gy in 2.5 Gy fractions or an equivalent regiment, has made significant inroads among North American GU experts in the treatment of prostate cancer, as more than 40\% of experts recommended hypofractionated EBRT as their preferred EBRT treatment modality. Nevertheless, 55\% of experts still consider conventionally fractionated EBRT as an unchallenged standard of care. Physicians who embrace a shorter treatment modality (brachytherapy), despite possible increase in acute toxicity - also tend to support hypofractionated EBRT. The relative- ly even duality regarding conventional versus hypofractionated treatment recommendation for intermediate - risk prostate cancer despite the four randomized trials already published on this topic (6-9) speaks to the issue that randomized trials do not necessarily change the standard of care, particularly in the United States, and a significantly longer follow-up is required; this duality is reflected in the most updated clinically localized prostate cancer guidelines published jointly by the American Urological Association, American Society for Radiation Oncology (ASTR0), and the Society of Urologic Oncology $(15,16)$. Hypofractionation in breast cancer similarly was adopted in other countries much sooner than in the United States, where ASTRO consensus statements, educational sessions and even direct advertisement to patients regarding hypofractionated options and their non - inferiority, led to final acceptance of hypofractionation as a new standard of care. It is unclear whether reimbursement system in the U.S. is partially responsible for a slower update of shorter treatment courses. Limitations of this study are relatively small sample size, despite an impressive (but still below fifty percent) response rate, inability to capture a full range of options due to multiple choice format, and a lack of granularity in addressing the impact of racial demographic of patients being treated (17). Furthermore, the absence of decade - long toxicity and outcome data comparing conventional versus moderate hypofractionation provides an uncertainty of outcomes beyond the five years of currently published results (6-11).

In conclusion, there is currently a nearly even split between radiation oncology experts in North America recommending conventionally fractionated vs moderately hypofractionated EBRT for patients with prostate cancer, based on dramatically different interpretation of results of 4 randomized clinical trials. Longer follow-up of these trials may impact the balance, while national care and reimbursement policies may influence the accepted standard of care. 
Table 1 - Summary of the four randomized clinical trials comparing hypofractionation (H-RT) with conventional fractionation (C-RT) for prostate cancer (OS = overall survival; DFS = disease-free survival; RFS = relapse-free survival; GU = genitourinary; GI = gastrointestinal; $\mathbf{C l}=$ confidence interval).

\begin{tabular}{|c|c|c|c|c|c|c|}
\hline Trial & Hypofractionation regimen & $\begin{array}{l}\text { Follow-up } \\
\text { duration }\end{array}$ & Location & $\begin{array}{c}\text { Differences in } \\
\text { OS or DFS }\end{array}$ & $\begin{array}{c}\text { Differences } \\
\text { in GU toxicity } \\
\text { between } \\
\text { modalities }\end{array}$ & $\begin{array}{c}\text { Differences } \\
\text { in Gl toxicity } \\
\text { between } \\
\text { modalities }\end{array}$ \\
\hline $\begin{array}{l}\text { RTOG } \\
0415 \\
(7)\end{array}$ & 2.5 Gy x 28 & 5 years & USA & No & $\begin{array}{l}\text { No } \\
\text { (late GU trended } \\
\text { toward favoring } \\
\text { C-RT: } p=0.06 \text { ) }\end{array}$ & $\begin{array}{c}\text { Yes } \\
\text { (late GI: } \\
\mathrm{p}=0.002 \\
\text { favored C-RT) }\end{array}$ \\
\hline $\begin{array}{l}\text { CHHiP } \\
\text { (8) }\end{array}$ & $\begin{array}{l}3 \text { Gy x } 20 \\
3 \text { Gy x } 19\end{array}$ & 5 years & $\begin{array}{l}\text { UK, Ireland, } \\
\text { Switzerland, } \\
\text { New Zealand }\end{array}$ & No & No & $\begin{array}{c}\text { Yes (acutely } \\
\text { favoring C-RT; } \\
\text { none by week } \\
\text { 18) }\end{array}$ \\
\hline $\begin{array}{l}\text { PROFIT } \\
\text { (6) }\end{array}$ & 3 Gy $x 20$ & 5 years & $\begin{array}{c}\text { Canada, } \\
\text { Australia, France }\end{array}$ & No & $\begin{array}{c}\text { No (acutely; late } \\
\text { toxicity favored } \\
\text { H-RT) }\end{array}$ & $\begin{array}{c}\text { No (acutely; } \\
\text { late toxicity } \\
\text { favored H-RT) }\end{array}$ \\
\hline $\begin{array}{l}\text { HYPRO } \\
(9-11)\end{array}$ & 3.4 Gy x 19 & 5 years & Netherlands & No & $\begin{array}{l}\text { Yes (H-RT } \\
\text { inferior for } \\
\text { acute and } \\
\text { late grade 3+ } \\
\text { toxicity) }\end{array}$ & $\begin{array}{l}\text { Yes (H-RT } \\
\text { inferior for } \\
\text { acute but not } \\
\text { late grade 3+ } \\
\text { toxicity) }\end{array}$ \\
\hline
\end{tabular}

\begin{tabular}{|c|c|c|c|c|c|c|}
\hline Trial & $\begin{array}{l}\text { GU toxicity } \\
\text { (H-RT) }\end{array}$ & $\begin{array}{l}\text { GU toxicity } \\
\text { (C-RT) }\end{array}$ & $\begin{array}{l}\text { Gl toxicity } \\
\text { (H-RT) }\end{array}$ & $\begin{array}{l}\text { Gl toxicity } \\
\text { (C-RT) }\end{array}$ & $\begin{array}{l}\text { Disease control } \\
\quad(\mathrm{H}-\mathrm{RT})\end{array}$ & $\begin{array}{l}\text { Disease control } \\
\quad(C-R T)\end{array}$ \\
\hline $\begin{array}{l}\text { RTOG } \\
0415 \\
(7)\end{array}$ & $\begin{array}{c}\text { Early grade } \\
2-4 \mathrm{GU}= \\
147 / 545 \\
\text { Late grade } \\
2-4 \mathrm{GU}= \\
161 / 545\end{array}$ & $\begin{array}{c}\text { Early grade } \\
2-4 \mathrm{GU}= \\
145 / 534 \\
\text { Late grade } \\
2-4 \mathrm{GU}= \\
121 / 534\end{array}$ & $\begin{array}{c}\text { Early grade } \\
2-4 \mathrm{GI}= \\
58 / 545 \\
\text { Late grade } \\
2-4 \mathrm{Gl}= \\
121 / 545\end{array}$ & $\begin{array}{c}\text { Early } \\
\text { grade } \\
2-4 \mathrm{Gl}= \\
55 / 534 \\
\text { Late grade } \\
2-4 \mathrm{Gl}= \\
75 / 534\end{array}$ & $\begin{array}{c}86.3 \% \text { DFS (95\% Cl: 83.1- } \\
89.0)\end{array}$ & $\begin{array}{c}85.3 \% \text { DFS (95\% Cl: } 81.9- \\
88.1)\end{array}$ \\
\hline $\begin{array}{l}\text { CHHiP } \\
\text { (8) }\end{array}$ & $\begin{array}{c}\text { Early grade } \\
2-4 \mathrm{GU}= \\
46-49 \% \\
\text { Late grade } \\
2-4 \mathrm{GU}= \\
6.6-11.7 \%\end{array}$ & $\begin{array}{c}\text { Early grade } \\
2-4 \mathrm{GU}= \\
46 \% \\
\text { Late grade } \\
2-4 \mathrm{GU}= \\
9.1 \%\end{array}$ & $\begin{array}{c}\text { Early grade } \\
2-4 \mathrm{GI}= \\
38 \% \\
\text { Late grade } \\
2-4 \mathrm{Gl} \\
=11.3- \\
11.9 \%\end{array}$ & $\begin{array}{c}\text { Early } \\
\text { grade } 2-4 \\
\mathrm{Gl}=25 \% \\
\text { Late grade } \\
2-4 \mathrm{GI}= \\
13.7 \%\end{array}$ & $\begin{array}{l}\text { 85.9-90.6\% biochemical/ } \\
\text { clinical failure freedom }\end{array}$ & $\begin{array}{l}88.3 \% \text { biochemical } / \text { clinical } \\
\text { failure freedom }\end{array}$ \\
\hline
\end{tabular}




\begin{tabular}{|c|c|c|c|c|c|c|}
\hline $\begin{array}{l}\text { PROFIT } \\
\text { (6) }\end{array}$ & $\begin{array}{c}\text { Early grade } \\
2-4 \text { GU = } \\
185 / 608 \\
\text { Late grade } \\
2-4 \mathrm{GU}= \\
136 / 608\end{array}$ & $\begin{array}{c}\text { Early grade } \\
2-4 \text { GU = } \\
183 / 598 \\
\text { Late grade } \\
3-4 \text { GU = } \\
134 / 598\end{array}$ & $\begin{array}{c}\text { Early grade } \\
2-4 \mathrm{GI}= \\
99 / 608 \\
\text { Late grade } \\
2-4 \mathrm{GI}= \\
54 / 608\end{array}$ & $\begin{array}{c}\text { Early } \\
\text { grade } \\
2-4 \mathrm{Gl}= \\
62 / 598 \\
\text { Late grade } \\
2-4 \mathrm{Gl}= \\
83 / 598\end{array}$ & $85 \%$ DFS & $85 \%$ DFS \\
\hline $\begin{array}{l}\text { HYPRO } \\
(9-11)\end{array}$ & $\begin{array}{c}\text { Early grade } \\
2-4 \mathrm{GU}= \\
75 / 410 \\
\text { Late grade } \\
2-4 \mathrm{GU} \text { at } \\
\text { three years } \\
=163 / 395\end{array}$ & $\begin{array}{c}\text { Early grade } \\
2-4 \mathrm{GU}= \\
73 / 410 \\
\text { Late grade } \\
3-4 \mathrm{GU} \text { at } \\
\text { three years } \\
=151 / 387\end{array}$ & $\begin{array}{l}\text { Early grade } \\
2-4 \mathrm{GI}= \\
42 / 410 \\
\text { Late grade } \\
2-4 \mathrm{GI} \text { at } \\
\text { three years } \\
=86 / 395\end{array}$ & $\begin{array}{c}\text { Early } \\
\text { grade } \\
2-4 \mathrm{Gl}= \\
43 / 410 \\
\text { Late grade } \\
2-4 \mathrm{Gl} \\
\text { at three } \\
\text { years = } \\
68 / 387\end{array}$ & $\begin{array}{c}80.5 \% \text { five-year RFS }(95 \% \\
\text { CI: } 75.7-84.4)\end{array}$ & $\begin{array}{c}77.1 \% \text { five-year RFS }(95 \% \mathrm{Cl} \text { : } \\
71.9-81.5)\end{array}$ \\
\hline
\end{tabular}

Figure 1 - Default External Beam Radiation Therapy Fractionation used by North American genitourinary oncology expert radiation oncologists for treatment of a hypothetical patient with a favorable intermediate risk Prostate Cancer (Gleason $3+4$ ).

\section{Default EBRT fractionation for Gleason 3+4 PCa}

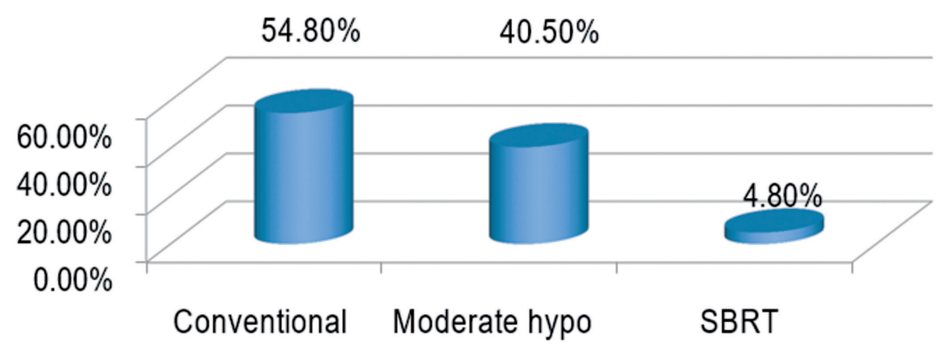

$\mathbf{P C a}=$ prostate cancer hypo $=$ hypofractionation 
Table 2 - Association between clinical practice recommendations and choice of default dose/fractionation for Gleason 3+4 prostate adenocarcinoma.

\begin{tabular}{|c|c|c|c|c|}
\hline Clinical Scenario & $\begin{array}{l}\text { Clinical Practice } \\
\text { Recommendation }\end{array}$ & $\begin{array}{c}\text { Conventional } \\
\text { Fractionation } \\
\text { (78 Gy in } 2 \mathrm{~Gy} \\
\text { fractions, } 79.2 \mathrm{~Gy} \text { in } \\
1.8 \text { Gy fractions, or } \\
\text { equivalent) }\end{array}$ & $\begin{array}{c}\text { Moderate } \\
\text { Hypofractionation } \\
\text { (70 Gy in } 2.5 \text { Gy fractions } \\
\text { or equivalent) }\end{array}$ & $P$ value \\
\hline \multirow{2}{*}{$\begin{array}{l}\text { Active surveillance recommendation } \\
\text { for Gleason } 6 \text { disease }\end{array}$} & Yes & $21(91.3 \%)$ & $17(100 \%)$ & 0.546 \\
\hline & No & $2(8.7 \%)$ & $0(0 \%)$ & \\
\hline \multirow{2}{*}{$\begin{array}{l}\text { Active surveillance recommendation } \\
\text { for Gleason } 3+4 \text { disease }\end{array}$} & Yes & $3(13.0 \%)$ & $4(23.5 \%)$ & 0.607 \\
\hline & No & $20(87.0 \%)$ & $13(76.5 \%)$ & \\
\hline \multirow[t]{2}{*}{ SBRT for oligometastatic lesions } & Yes & $18(78.3 \%)$ & $12(70.6 \%)$ & 0.837 \\
\hline & No & $5(21.7 \%)$ & $5(29.4 \%)$ & \\
\hline \multirow{2}{*}{$\begin{array}{l}\text { Treatment of pelvic lymph nodes in } \\
\text { localized high-risk prostate cancer }\end{array}$} & Rarely & $9(39.1 \%)$ & $4(23.5 \%)$ & 0.377 \\
\hline & Often & $14(60.9 \%)$ & $13(76.5 \%)$ & \\
\hline \multirow[t]{2}{*}{ Treatment of high-risk prostate cancer } & EBRT+ADT & $15(65.2 \%)$ & $7(41.2 \%)$ & 0.305 \\
\hline & $\begin{array}{c}\text { EBRT+ADT+ } \\
\text { brachytherapy boost }\end{array}$ & $8(34.8 \%)$ & $10(58.8 \%)$ & \\
\hline \multirow{2}{*}{$\begin{array}{l}\text { Believer in advanced-imaging (Novel } \\
\text { ligand-based PET imaging) }\end{array}$} & Yes & $14(60.9 \%)$ & $14(82.4 \%)$ & 0.137 \\
\hline & No & $9(39.1 \%)$ & $2(11.8 \%)$ & \\
\hline \multirow{3}{*}{$\begin{array}{l}\text { First choice for treatment of Gleason } 6 \\
\text { disease who desires intervention }\end{array}$} & Brachytherapy & $8(34.8 \%)$ & $12(70.6 \%)$ & 0.089 \\
\hline & EBRT & $5(21.7 \%)$ & $1(5.9 \%)$ & \\
\hline & No preference & $10(43.5 \%)$ & $4(23.5 \%)$ & \\
\hline
\end{tabular}

\section{CONFLICT OF INTEREST}

None declared.

\section{REFERENCES}

1. Deshmukh AA, Shirvani SM, Lal L, Swint JM, Cantor SB, Smith BD, et al. Cost-effectiveness Analysis Comparing Conventional, Hypofractionated, and Intraoperative Radiotherapy for Early-Stage Breast Cancer. J Natl Cancer Inst. 2017;109.
2. Pichon B, Campion L, Delpon G, Thillays F, Carrie C, Cellier $P$, et al. High-Dose Hypofractionated Radiation Therapy for Noncompressive Vertebral Metastases in Combination With Zoledronate: A Phase 1 Study. Int J Radiat Oncol Biol Phys. 2016;96:840-7.

3. Hafeez $\mathrm{S}$, McDonald F, Lalondrelle $\mathrm{S}$, McNair H, WarrenOseni K, Jones K, et al. Clinical Outcomes of Image Guided Adaptive Hypofractionated Weekly Radiation Therapy for Bladder Cancer in Patients Unsuitable for Radical Treatment. Int J Radiat Oncol Biol Phys. 2017;98:115-22. Erratum in: Int J Radiat Oncol Biol Phys. 2018;100:532-3. 
4. Malmström A, Grønberg BH, Marosi C, Stupp R, Frappaz $\mathrm{D}$, Schultz $\mathrm{H}$, et al. Temozolomide versus standard 6-week radiotherapy versus hypofractionated radiotherapy in patients older than 60 years with glioblastoma: the Nordic randomised, phase 3 trial. Lancet Oncol. 2012;13:916-26.

5. Walraven I, van den Heuvel M, van Diessen J, Schaake E, Uyterlinde W, Aerts J, et al. Long-term follow-up of patients with locally advanced non-small cell lung cancer receiving concurrent hypofractionated chemoradiotherapy with or without cetuximab. Radiother Oncol. 2016;118:442-6.

6. Catton CN, Lukka H, Gu CS, Martin JM, Supiot S, Chung PWM, et al. Randomized Trial of a Hypofractionated Radiation Regimen for the Treatment of Localized Prostate Cancer. J Clin Oncol. 2017;35:1884-90.

7. Lee WR, Dignam JJ, Amin MB, Bruner DW, Low D, Swanson GP, et al. Randomized Phase III Noninferiority Study Comparing Two Radiotherapy Fractionation Schedules in Patients With Low-Risk Prostate Cancer. J Clin Oncol. 2016;34:2325-32.

8. Dearnaley D, Syndikus I, Mossop H, Khoo V, Birtle A, Bloomfield D, et al. Conventional versus hypofractionated high-dose intensity-modulated radiotherapy for prostate cancer: 5-year outcomes of the randomised, non-inferiority, phase 3 CHHiP trial. Lancet Oncol. 2016;17:1047-60. Erratum in: Lancet Oncol. 2016;17:e321.

9. Incrocci L, Wortel RC, Alemayehu WG, Aluwini S, Schimmel E, Krol S, et al. Hypofractionated versus conventionally fractionated radiotherapy for patients with localised prostate cancer (HYPRO): final efficacy results from a randomised, multicentre, open-label, phase 3 trial. Lancet Oncol. 2016;17:1061-9.

10. Aluwini S, Pos F, Schimmel E, van Lin E, Krol S, van der Toorn PP, et al. Hypofractionated versus conventionally fractionated radiotherapy for patients with prostate cancer (HYPRO): acute toxicity results from a randomised noninferiority phase 3 trial. Lancet Oncol. 2015;16:274-83. Erratum in: Lancet Oncol. 2015;16:e105.

11. Aluwini S, Pos F, Schimmel E, Krol S, van der Toorn PP, de Jager $\mathrm{H}$, et al. Hypofractionated versus conventionally fractionated radiotherapy for patients with prostate cancer (HYPRO): late toxicity results from a randomised, noninferiority, phase 3 trial. Lancet Oncol. 2016;17:464-74.
12. Harris PA, Taylor R, Thielke R, Payne J, Gonzalez N, Conde JG. Research electronic data capture (REDCap)--a metadatadriven methodology and workflow process for providing translational research informatics support. J Biomed Inform. 2009;42:377-81.

13. Hegemann NS, Guckenberger M, Belka C, Ganswindt U, Manapov F, Li M. Hypofractionated radiotherapy for prostate cancer. Radiat Oncol. 2014;9:275.

14. Nass SJ, Balogh E, Mendelsohn J. A National Cancer Clinical Trials Network: recommendations from the Institute of Medicine. Am J Ther. 2011;18:382-91.

15. Sanda MG, Cadeddu JA, Kirkby E, Chen RC, Crispino T, Fontanarosa J, et al. Clinically Localized Prostate Cancer: AUA/ASTRO/SUO Guideline. Part I: Risk Stratification, Shared Decision Making, and Care Options. J Urol. 2018;199:683-90.

16. Sanda MG, Cadeddu JA, Kirkby E, Chen RC, Crispino T, Fontanarosa J, et al. Clinically Localized Prostate Cancer: AUA/ASTRO/SUO Guideline. Part II: Recommended Approaches and Details of Specific Care Options. J Urol. 2018;199:990-7.

17. McClelland S 3rd, Sandler KA, Degnin C, Chen Y, Mitin T. Active Surveillance for Low and Intermediate Risk Prostate Cancer: Opinions of North American Genitourinary Oncology Expert Radiation Oncologists. Clin Genitourin Cancer. 2018;16:e323-5.
Correspondence address:

Timur Mitin, MD, PhD

Department of Radiation Medicine Oregon Health \& Science University 3181 SW Sam Jackson Park Rd, L337

Portland, OR, 97239-3098, U.S.A

Fax: +1 503 346-0237

E-mail:mitin@ohsu.edu 


\section{Confidential}

\section{Advanced imaging techniques in prostate cancer}

Please complete the survey below. It is 4 pages long and should take approximately 5 minutes to finish.

Thank you very much for your contribution!

Are you actively practicing clinical oncology?

$\bigcirc$ Yes $\bigcirc$ No

Is genitourinary oncology your primary focus?

$\bigcirc$ Yes $\bigcirc$ No

What is your specialty?

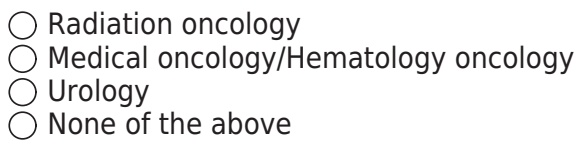

How many years has it been since you completed training (residency/oncology fellowship)?
$0-4$ years
5-10 years
$11-20$ years
$>>20$ years
Still in training 


\section{Confidential}

Where did you complete your training?

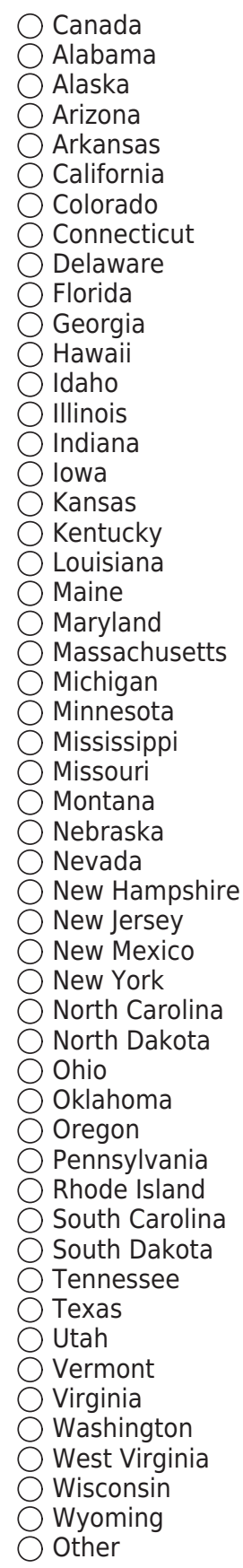


Confidential

Where do you primarily practice?

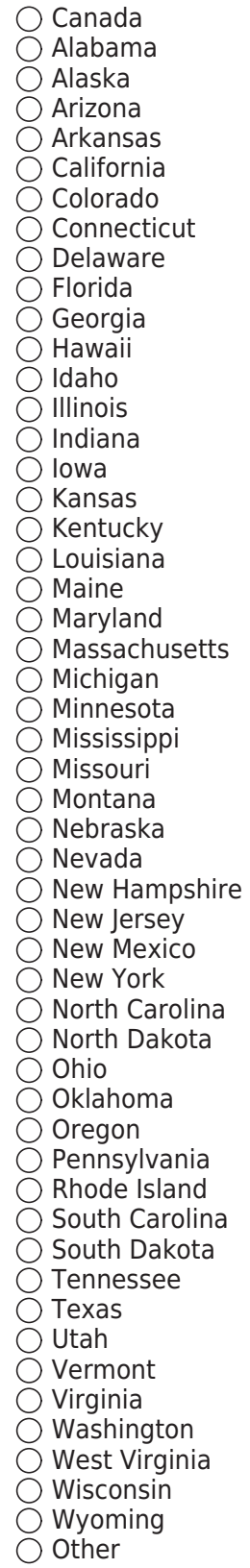

How would you best describe your primary practice setting?

Academic/university

$\bigcirc$ Hospital-based, no academic/university affiliation

Free-standing, no academic/university affiliation

Government employed, such as VA, military, or government-run facility Other 


\section{Confidential}

Page 4 of 8

How many patients with prostate cancer do you see in consultation per month on average?

0-4

5-9

10-14

15-20

$>20$ 


\section{Confidential}

\section{Please tell us more about your practice characteristics.}

For this section, check all that apply.

I often recommend active surveillance for patients with Gleason 6 disease.

I often recommend active surveillance for patients with Gleason 3+4=7 disease.

For patients with oligometastatic disease, I would consider offering stereotactic body radiation therapy to the oligometastatic lesion outside of a clinical trial.

Please select one of the following options.

Most patients I see in clinic present with intact prostate for discussion of definitive treatment.

$\bigcirc$ Most patients I see in clinic present after prostatectomy for a discussion of adjuvant or salvage radiation.

$\bigcirc$ I see an even balance of patients with intact prostate and those who are post-prostatectomy.

Please select one of the following options.

As a general rule for patients with high risk features, I recommend adjuvant radiation after surgery.

As a general rule for patients with high risk features, I recommend observation and early salvage radiation if PSA rises.

Please select one of the following options.

For localized high risk prostate cancer, I treat pelvic lymph nodes rarely.

For localized high risk prostate cancer, I treat pelvic lymph nodes often.

Do you consider yourself an expert brachytherapist?

Yes

No

For patients with Gleason 6 disease who desire treatment, with no baseline urinary symptoms and a 40cc prostate, which would you consider your first choice for treatment?

External beam radiation

Brachytherapy

$\bigcirc$ Either external beam or brachy (no preference)

For patients with localized high risk disease, with no baseline urinary symptoms and a $40 \mathrm{cc}$ prostate, which would you consider your first choice for treatment?

External beam radiation with ADT (androgen deprivation therapy)

$\bigcirc$ External beam radiation with ADT and brachytherapy boost

What is your current practice with regard to digital rectal examinations (DRE)? (Check all that apply)

$\square$ I routinely perform DRE before treatment

I routinely perform DRE at follow-up visits

I never perform DRE

I believe DRE will change management

I do not believe DRE will change management

What do you consider the default EBRT dose and fractionation for Gleason $3+4$ prostate adenocarcinoma?

Conventional fractionation: 78 Gy in 2 Gy fractions, 79.2 Gy in 1.8, or equivalent

Moderate hypofractionation: 70 Gy in 2.5 Gy fractions or equivalent

SBRT/Radical hypofractionation: 5-12 fractions or equivalent 
Are you aware that the NCCN recommends consideration of C-11 choline PET, but not PSMA PET for patients with prostate cancer?

Y Yes

$\bigcirc$ No

The NCCN recommends considering C-11 PET in the following scenarios:

- In the setting of detectable PSA after prostatectomy

- Biochemical failure after definitive radiation

- In MO patients on androgen deprivation therapy with a rising PSA.

What are your thoughts on these recommendations?

I agree with the NCCN recommendations.

The NCCN should recommend the use of C-11 PET in more scenarios than those listed above (specify)

$\bigcirc$ The NCCN should recommend considering C-11 PET in some, but not all of the above scenarios (specify)

The NCCN should not recommend considering C-11 PET at all because there is not enough evidence to support its use in routine practice.

Please specify

Do you think that the NCCN should recommend consideration of PSMA PET?

The NCCN should recommend consideration of PSMA PET in the same scenarios as C-11 PET.

The NCCN should recommend consideration of PSMA PET in more scenarios than C-11 PET (specify).

The NCCN should recommend consideration of PSMA PET in some, but not all of the same scenarios as C-11 PET (specify).

The NCCN should not recommend consideration of PSMA PET because there is not enough evidence to support its use in routine practice.

Please specify

Regarding the comparison of C-11 PET to PSMA PET, select the answer which best describes your opinion.

C-11 PET has better efficacy than PSMA PET.

PSMA PET has better efficacy than C-11 PET.

C-11 PET and PSMA PET have the same level of efficacy.

There is not enough data to know whether C-11 PET or PSMA PET is more effective.

Which of the following imaging studies are available at your practice (or at an affiliated facility)?

C-11 PET

$\bigcirc$ PSMA PET

Both

$\bigcirc$ Neither

What is your current practice with regard to the new imaging modalities PSMA PET and C-11 PET?

I routinely order them for my patients and use the results to guide treatment decision-making.

$\bigcirc$ I have ordered them on rare occasion for my patients and used the results to guide treatment decision-making.

I do not order them, but if a patient already has results at the time I see them, I will use the results to guide treatment decision-making.

I do not order them and do not use the results to guide treatment decision-making. 


\section{Confidential}

What is your current practice with regard to the new imaging modalities PSMA PET and C-11 PET?

I often refer patients to centers capable of performing one of these tests.

On rare occasions I have referred my patients to centers capable of performing one of these tests.

I do not refer them, but if a patient already has results at the time I see them, I will use the results to guide treatment decision-making.

I do not refer them and do not use the results to guide treatment decision-making.

What is the primary reason you do not use results from PSMA PET or C-11 PET to guide treatment decision-making?

There is not enough data to guide usage of these tests.

I do not believe these tests are effective.

I lack personal experience using these tests.

Other (specify)

Please specify 


\section{Confidential}

\section{For each of the following patient scenarios, please enter the lowest PSA value at which you would order a C-11 PET or PSMA PET. Enter 0 for "never."}

Gleason $4+5=9$, post-prostatectomy, consecutively rising PSA

Gleason 4+3=7, post-prostatectomy, consecutively rising PSA

Gleason 10, T3, intact prostate, pre-treatment

Gleason $4+3=7, T 2$, intact prostate, pre-treatment

Gleason 10, T3, post-definitive radiation

Gleason $4+3=7, T 2$, post-definitive radiation

In a patient with newly diagnosed cT2 Gleason 9 prostate cancer with a PSA of $200 \mathrm{ng} / \mathrm{mL}$, who has no evidence of bone metastases by nuclear bone scan and abdominopelvic CT, which of the following would you consider for further workup?

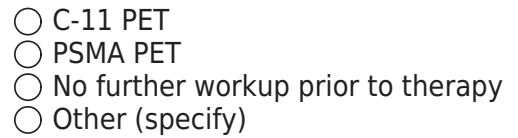

Please specify

What are the reasons you do not order PSMA PET or C-11 PET more frequently? (Check all that apply)

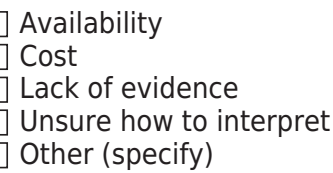

Please specify 